\title{
Management of pyelovesical bypass device stones
}

Ahmad Almarzouq, MD, MSc; Sero Andonian, MD, MSc, FRCSC, FACS

Department of Urology, McGill University Health Centre, Montreal, QC, Canada

Cite as: Can Urol Assoc J 2018 Feb. 6; Epub ahead of print. http://dx.doi.org/10.5489/cuaj.4857

Published online February 6, 2018

$* * *$

\section{Introduction}

Pyelovesical bypass devices or artificial ureters have been described as a last resort in patients with long ureteral strictures that fail traditional endoscopic and open repair. Herein, we describe a 52-year old female who had a Detour (Coloplast, Humlebaek, Denmark) pyelovesical bypass device inserted after an iatrogenic ischemic injury to the distal two-thirds of the left ureter during pelvic surgery for recurrent endometrial stromal sarcoma. Six months after placement of the device, she presented with gross hematuria and recurrent urinary tract infections and was found to have encrustation of the distal silicone tip of the Detour device within the bladder. This was managed with resection of the distal silicone tip and flexible ureteroscopy with Holmium laser lithotripsy. Despite suppressive antibiotic therapy and medical therapy for hypercalciuria, she presented four years later with intraluminal encrustations in the proximal end of the device. This was successfully managed with flexible ureteroscopy with Holmium laser lithotripsy. Therefore, this case illustrates the feasibility of flexible ureteroscopy and Holmium laser lithotripsy of Detour device encrustations as long as the device is not kinked and it allows the passage of the flexible ureteroscope up to the calcifications. In addition, patients contemplating insertion of such devices should be counselled regarding the risk of recurrent infections and encrustations. 


\section{Almarzouq and Andonian Pyelovesical bypass device stones}

\section{Case report}

We present a 52-year-old female who had undergone total abdominal hysterectomy for endometrial stromal cell sarcoma. Four years later, she underwent laparoscopic resection of recurrence invading the left pelvic wall. One week post-operatively, she presented with exsanguinating hemorrhage and was taken for an urgent open exploration where the source of the bleeding was found to be from the left internal iliac vein. Furthermore, it was discovered that she had necrosis of the distal two-thirds of the left ureter in addition to a 4cm cystotomy in the left bladder wall. The general surgeon resected the necrotic rectum and created an end sigmoid colostomy. Due to a hostile abdomen and hemodynamic instability (6 units of PRBC for an estimated blood loss of 1.5L), the decision was made to tie the ureter and insert a nephrostomy tube post-operatively. The bladder wall defect was repaired in 2 layers and was confirmed to be water tight. The patient recovered well and subsequently underwent resection of lung metastasis without any other sarcoma recurrences. Because of the small bladder capacity and necrosis of the distal two-thirds of the left ureter, it was decided that the best option would be to place a subcutaneous pyelovesical bypass graft (Detour ${ }^{\mathrm{TM}}$ from Coloplast, Humlebaek, Denmark) to improve her quality of life, since she already had a colostomy in addition to her left nephrostomy.

Six months following the insertion of the Detour ${ }^{\mathrm{TM}}$ device, the patient presented with gross hematuria and recurrent urinary tract infections (UTIs). CT scan showed calcifications on the distal silicone tip of the Detour device which was later confirmed with cystoscopy (Figure 1A). There were no other calcifications. The encrusted Detour ${ }^{\mathrm{TM}}$ was managed cystoscopically by cutting the $2 \mathrm{~cm}$ intra-vesical silicone portion of the device in addition to Holmium laser lithotripsy of the intraluminal calcifications with a flexible ureteroscope (Figure 1B). Stone analysis revealed calcium phosphate and metabolic stone work-up showed hypercalciuria which was treated with hydrocholorothiazide-amiloride. The recurrent UTIs were treated with prophylactic nitrofurantoin and mandelamine. She had closure of her colostomy and repair of her ventral hernia. Four years after resection of the distal tip of the Detour ${ }^{\mathrm{TM}}$ device, she presented with encrustations in the proximal tip of the Detour ${ }^{\mathrm{TM}}$ device causing hydronephrosis (Figure 1C). A second flexible ureteroscopy was performed and intraluminal calcifications were pulverized using Holmium laser leading to successful clearance of the stone burden and reversal of the hydronephrosis (Figure 1D).

\section{Discussion}

Iatrogenic ureteric injuries are uncommon, but when they do occur, they can have devastating consequences depending on the extent and mode of injury ${ }^{1,2}$. Ureteric injuries are encountered following gynecological surgery, namely hysterectomy (vaginal and abdominal), colorectal surgery, namely lower anterior resection and abdomino-perineal resection and urological surgery. Most common site of injury is the distal ureter ${ }^{2}$. In our case, the injury was extensive and there was loss of approximately two thirds of the ureteric length. This rendered most repair 
approaches unsuitable and only ileal interposition or auto-transplantation would have been applicable. These 2 options would have had a high chance of failure if performed given the hostile surgical field and hemodynamic instability at the time of injury. Therefore, a temporizing nephrostomy was inserted with view of repairing the ureter later, but given the poor prognosis of the patient at the time, the decision was made to insert a subcutaneous pyelovesical bypass device to bypass the hostile pelvis.

Since the advent of pyelovesical bypass devices in the late nineties, they have been used exclusively to palliate patients with terminal malignancies or to salvage renal transplants that failed open or endoscopic repair of uretero-neo-vesical strictures ${ }^{3,4}$. Early results from Europe and Canada have shown excellent outcomes in both quality of life and graft salvage rates 5,6 . However, reports of complications increased with longer follow-up ${ }^{7,8}$. In this particular case, the original intent was to address the patient's quality of life (colostomy and nephrostomy) as her prognosis was poor with metastatic uterine sarcoma. However, she did not have any recurrence of her metastatic sarcoma after lung resection and presented with recurrent UTIs leading to recurrent encrustations of her pyelovesical bypass graft. These encrustations were managed successfully with ureteroscopy and Holmium laser lithotripsy. Previous attempts have failed ureteroscopic management of Detour encrustations ${ }^{9}$. This case demonstrates the feasibility of flexible ureteroscopy and Holmium laser lithotripsy of encrustations since there were no kinks in the Detour device.

In conclusion, pyelovesical devices should be reserved as a last resort option to palliate patients with chronic nephrostomies. In addition, patients should be advised of the long-term risks of recurrent infections and encrustations. Should encrustations occur, these could be successfully managed with flexible ureteroscopy and Holmium laser lithotripsy as long as there are no kinks in the device. 


\section{Almarzouq and Andonian Pyelovesical bypass device stones}

\section{References}

1- Selzman AA, Spirnak JP. Iatrogenic ureteral injuries: a 20-year experience in treating 165 injuries. J Urol. 1996;155(3):878-81.

2- Delacroix SE Jr, Winters JC. Urinary tract injures: recognition and management. Clin Colon Rectal Surg. 2010;23(2):104-12.

3- Desgrandchamps F, Paulhac P, Fornairon S et al. Artificial ureteral replacement for ureteral necrosis after renal transplantation: report of 3 cases. J Urol. 1998;159(6):1830-2.

4- Andonian S, Zorn KC, Paraskevas S et al. Artificial ureters in renal transplantation. Urology. 2005;66(5):1109-09.

5- Desgrandchamps F, Leroux S, Ravery V et al. Subcutaneous pyelovesical bypass as replacement for standard percutaneous nephrostomy for palliative urinary diversion: prospective evaluation of patient's quality of life. J Endourol. 2007;21(2):173-6.

6- Azhar RA, Hassanain M, Aljiffry M et al. Successful salvage of kidney allografts threatened by ureteral stricture using pyelovesical bypass. Am J Transplant. 2010;10(6):1414-9.

7- Janitzky A, Borski J, Porsch M et al. Long-term results for subcutaneous Detour ${ }^{\circledR}$ prosthesis for ureteral obstruction: experiences of implantation, aftercare and management of complications. Urologe A. 2012;51(12):1714-21.

8- Yazdani M, Javid A, Mohammadi Sichani M et al. Artificial Ureter in Patients with Extensive Ureteral Damage. Urol J. 2017;14(3):3091-3093.

9- Wilhelm K, Schultze-Seemann W, Miernik A. Complete Occlusion of a Subcutaneous Pyelovesical Bypass Graft (Detour® System) Caused by Uric Acid Stone Formation. Urol Int. 2017;98(4):483-485. 


\section{Figures and Tables}

Fig. 1. (A) Axial images of the distal tip of the device with encrustations. (B) Axial images of the distal tip of the device post resection and Holmium laser fragmentation. (C) Axial images of the left Renal pelvis illustrating encrustations at the proximal tip of the device causing obstruction. (D) Axial images of the left renal pelvis illustrating clearance of the intra-luminal encrustations and resolution of the hydronephrosis post ureteroscopy and Holmium laser fragmentation.

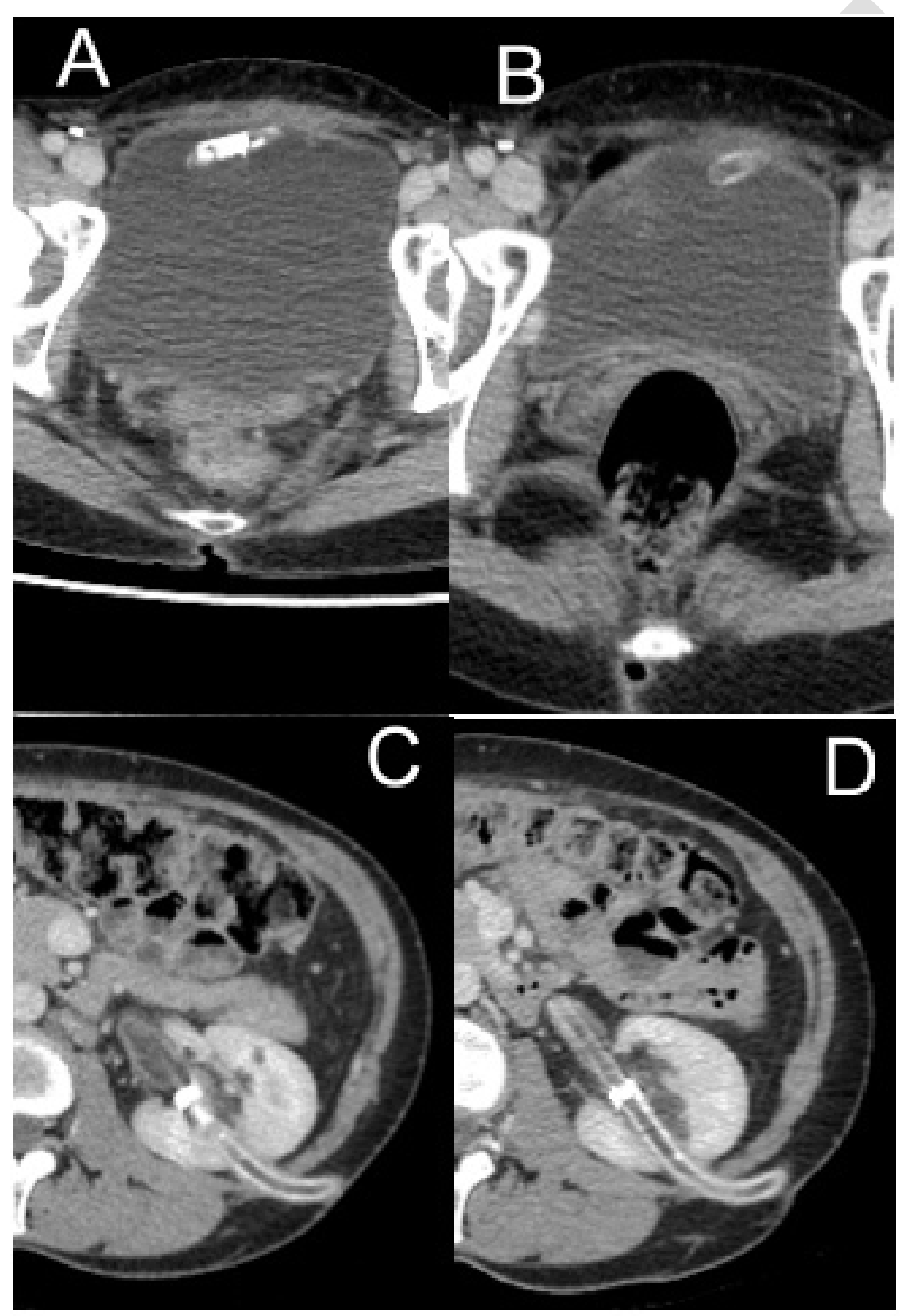

